\title{
Frontiers of beam diagnostics in plasma accelerators: Measuring the ultra-fast and ultra-cold
}

\author{
A. Cianchi, ${ }^{1, a), b)}$ M. P. Anania ${ }^{2}$ F. Bisesto, ${ }^{2}$ E. Chiadroni, ${ }^{2}$ A. Curcio, ${ }^{2}$ M. Ferrario, ${ }^{2}$ \\ A. Giribono, ${ }^{2}$ A. Marocchino, ${ }^{2}$ R. Pompili, ${ }^{2}$ J. Scifo, ${ }^{2}$ V. Shpakov, ${ }^{2}$ C. Vaccarezza, ${ }^{2}$ F. Villa, ${ }^{2}$ \\ A. Mostacci, ${ }^{3}$ A. Bacci, ${ }^{4}$ A. R. Rossi, ${ }^{4}$ L. Serafini, ${ }^{4}$ and A. Zigler ${ }^{5}$ \\ ${ }^{1}$ University of Rome Tor Vergata and INFN, Via della Ricerca Scientifica 1, 00133 Roma, Italy \\ ${ }^{2}$ INFN-LNF, via E. Fermi 40, 00044 Frascati, Italy \\ ${ }^{3}$ University La Sapienza of Roma, via Antonio Scarpa 24, 00133 Roma, Italy \\ ${ }^{4}$ INFN-Milano, Via Celoria 16, 20133 Milano, Italy \\ ${ }^{5}$ Racah Institute of Physics, Hebrew University, 91904 Jerusalem, Israel
}

(Received 30 November 2017; accepted 15 February 2018; published online 28 March 2018)

\begin{abstract}
Advanced diagnostics are essential tools in the development of plasma-based accelerators. The accurate measurement of the quality of beams at the exit of the plasma channel is crucial to optimize the parameters of the plasma accelerator. 6D electron beam diagnostics will be reviewed with emphasis on emittance measurement, which is particularly complex due to large energy spread and divergence of the emerging beams, and on femtosecond bunch length measurements. Published by AIP Publishing. https://doi.org/10.1063/1.5017847
\end{abstract}

\section{INTRODUCTION}

Advancement in particle physics has always been linked with the availability of particle beams of ever increasing energy or intensity. However, nowadays accelerators are widely used in a very large spectrum of applications, from light sources to medical and industrial applications. Plasmabased concepts presently offer the highest gradient acceleration compared to other novel acceleration techniques like highfrequency W-band metallic RF structures, ${ }^{1}$ dielectric wakefield structures $^{2}$ or direct laser acceleration. ${ }^{3}$ This revolutionary change permits to avoid metallic or dielectric structure damage problems encountered in high-gradient operation. It can allow building high energy compact machines in a space reduced by a huge factor, between 10 and 100 .

Laser beams (laser wakefield accelerator, LWFA) or charged particle beams (particle wakefield accelerator, PWFA) may be adopted to excite space-charge oscillations in plasma. The resulting fields can be used for particle acceleration and focusing.

The EuPRAXIA ${ }^{4}$ (European Plasma Research Accelerator with eXcellence In Applications) project and foreseen facility is expected to be the first Research Infrastructure devoted to establish the scientific and technological basis required to build a compact and cost effective high energy (up to $5 \mathrm{GeV}$ ) machine based on plasma accelerator technology.

Currently, there are different schemes proposed and there is not yet a decision if this machine will be based on LWFA or PWFA. In terms of diagnostics, we noticed that there is not so much difference between the two schemes. In both cases, the need to remove the driver, being a high power laser or an electron beam, prevents to place diagnostics just after the plasma interaction. Also, the high beam divergence,

\footnotetext{
Note: Paper YI3 3, Bull. Am. Phys. Soc. 62, 405 (2017)

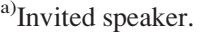

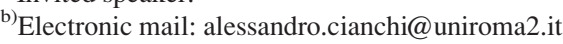

and likely the large energy spread, force the capture of the beam with proper optics as soon as possible. Therefore, it is very difficult to foresee in both cases, a diagnostics device between the plasma stage and the capture optics. After that, we do not expect significant differences between beams produced with different schemes and so we can consider to implement the same diagnostics for both schemes.

In the framework of the realization of a Conceptual Design Report for this project, we faced for the first time the problems to design diagnostics for a real big plasma machine, and not just simply for a small proof of principle experiment. The requests for plasma accelerators are quite tight especially in terms of resolution. Time resolution must be on the order of few fs, the transverse emittance resolution better than $1 \mathrm{~mm}$-mrad, the charge must be discriminate at the level of $\mathrm{pC}$, and the trajectory must be known at $\mu \mathrm{m}$ level. Last but not the least, all the diagnostics must be very compact, using as a small as possible longitudinal space along the accelerators. It results in a complete redesign of even conventional diagnostics.

This paper is not a review of the existing diagnostics techniques but, starting from the state of the art, it wants to emphasize the peculiarity of the plasma acceleration, and the challenges in measuring such kind of beams, giving also information about our developments still in progress. We will concentrate on the transverse diagnostics in Sec. II, focusing on an innovative technique, especially for single shot diagnostics. In Sec. III, we will analyze the longitudinal diagnostics for fs bunch length measurements.

\section{TRANSVERSE DIAGNOSTICS}

There are two main measurements for transverse diagnostics: emittance and envelope. The envelope is very important in order to properly match the beam along the machine comparing the measured dimensions with the simu-

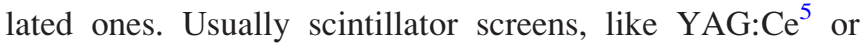


Optical Transition Radiation (OTR) monitors are in use for such a task, retrieving the $2 \mathrm{D}$ beam image in a single shot. In particular, YAG screens are implemented when the beam charge is below few tens of $\mathrm{pC}$, due to their better photon yield.

The emittance measurement can be performed for the main linac with very well-known techniques, like quadrupole scan and multiple screens. ${ }^{6}$ However, for plasma accelerated beams, the inherent shot by shot instabilities, with high beam divergence, and the need to separate driver and witness, prevent the use of such a diagnostic just after the plasma channel. In particular, the large energy spread (usually above \% level) is a serious drawback. Its value must be also kept as low as possible because, following Ref. 7, even the 6D rms normalized emittance is not preserved in a drift with energy spread, and so the measurement of the emittance is strongly dependent on the measurement position. This point is usually neglected but it deeply impacts the beam quality. We recall that the total normalized emittance squared is

$$
\varepsilon_{n}^{2}=\langle\gamma\rangle^{2} \sigma_{\varepsilon}^{2}\left\langle x^{2}\right\rangle\left\langle x^{\prime 2}\right\rangle+\langle\beta \gamma\rangle^{2}\left(\left\langle x^{2}\right\rangle\left\langle x^{\prime 2}\right\rangle-\left\langle x x^{\prime}\right\rangle^{2}\right),
$$

where $\gamma$ is the usual relativistic factor, $\beta$ is the ratio of the speed of the particle to the speed of light, and $\sigma_{\varepsilon}$ is the percentage energy spread.

Due to the presence of a non negligible energy spread, and being the divergence term usually on mrad, the first term will be the leading one after some drift. At this point, the normalized emittance has grown significantly, spoiling the beam properties.

Recently, the concept of chromatic length was introduced, ${ }^{8}$ defined as the distance where the emittance grows by a factor $\sqrt{2}$ as

$$
L_{C}=\frac{\sigma_{x}}{\sigma_{x}^{\prime} \sigma_{E}}
$$

where $\sigma_{x}$ is the rms beam size, $\sigma_{x}^{\prime}$ is the rms beam divergence, and $\sigma_{E}$ is the relative rms energy spread at plasma extraction. In a conventional accelerator, $\mathrm{L}_{C}$ is usually longer than the whole machine, while in plasma accelerators, depending on the value of the energy spread, $\mathrm{L}_{C}$ could be in a range between few centimeters and few meters. To overcome this problem, the only solution is a fast capture of this beam and a mitigation of the energy spread, even at the cost of some charge reduction.

For these reasons, we consider the use of a different approach in order to measure the beam parameters. We plan to measure them inside the plasma channel, and only after the machine optics devoted to capture the beam, to separate the driver and witness, and eventually to reduce the beam energy spread. So, we do not foresee any measurement just after the plasma channel.

The measurement inside the plasma can be performed by means of betatron radiation. The diagnostics based on betatron radiation ${ }^{9}$ has been developed in recent years in several laboratories, relying on the measurement of the spectrum, (for instance, among the other see Ref. 10) or on the diffraction from a knife edge. ${ }^{11}$
However, these systems were able to measure just the beam profile and divergence, neglecting the correlation term. Only recently, we developed a new algorithm in order to retrieve the correlation term. ${ }^{12}$ Using the simultaneous measurement of the electron and radiation energy spectrum together with the plasma density, it is possible to have a reconstruction of the whole phase space. This measurement relies on some approximation on the initial phase space of the particles, because it was performed on a beam produced by self-injection mechanics. If the beam is externally injected inside the plasma, the knowledge of the initial 6D phase space removes this ambiguity also.

In Fig. 1 is reported a reconstructed phase space with this technique. Laser parameters: energy $1 \mathrm{~J}$, pulse duration 30 fs $(\mathrm{FWHM}), 10 \mu \mathrm{m}$ diameter focus, $\mathrm{a}_{0} \approx 4.4$, and plasma density $=(8 \pm 1) 10^{18} \mathrm{~cm}^{-3}$.

This algorithm is based on the reconstruction of the beam 1D profile rather than just the beam size. Also, the full $2 \mathrm{D}$ beam profile characterization has also been shown to be possible to be measured using the correlation between spectrum and angle. ${ }^{13}$

In order to collect the betatron radiation, sooner or later, we have to separate the radiation from the electron beam, with a dipole. Unfortunately, the bending of the beam produces synchrotron radiation, and its spectrum can overlap with the betatron radiation.

We observe that usually the betatron radiation is much stronger with respect to synchrotron radiation, and there is the additional degree of freedom of the bending angle. The possibility to increase the magnetic field allows moving the peak of the synchrotron radiation at higher frequency, resulting in a better separation with betatron radiation.

In Fig. 2, we reported a comparison between the spectrum of the betatron radiation of $30 \mathrm{pC}$ beam accelerated up to $1 \mathrm{GeV}$ inside a plasma and the synchrotron radiation produced by a bending magnet. The details of the simulation are given in the caption.

However, an open problem is related to the separation between the betatron radiation coming from the witness and from the driver in the beam driven scheme. In this case, the driver contains much more charge with respect to the witness and so only a clear energy separation of the two spectra can solve the problem. Obviously in the case of external injection, this problem disappears.

After the capture optics, another single shot emittance measurement is needed in order to match the beam to an undulator. In our opinion, there is not yet a clear solution to this problem. Pepper pot like techniques ${ }^{14}$ are not easy to be

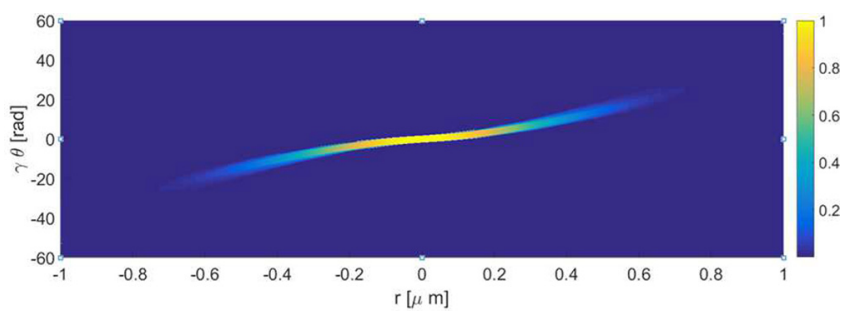

FIG. 1. Reconstructed phase space with betatron radiation from a selfinjected electron beam. ${ }^{12}$ 


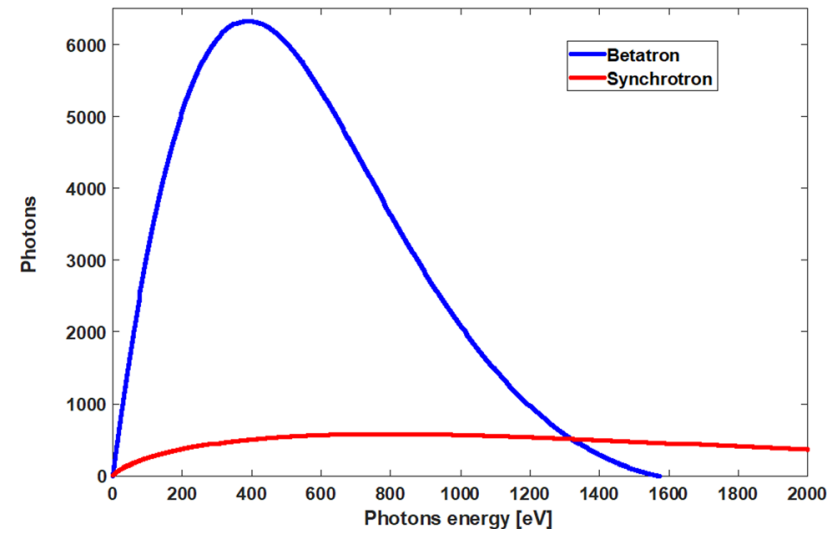

FIG. 2. Comparison between Synchrotron radiation emitted by a bending magnet and Betatron radiation. Beam charge $30 \mathrm{pC}$, energy $1 \mathrm{GeV}$, plasma density $2 \times 10^{16} \mathrm{~cm}^{-3}$, magnet field $1.5 \mathrm{~T}$, and radius of curvature $2.2 \mathrm{~m}$.

implemented at high energy, because to clearly separate the beamlets, a very tick target is needed. But the increase in the thickness reduces the angular acceptance of the beam, resulting in a likely cut of the phase space. However, the pepper pot suffers also for a problem related to the sampling nature of this measurement, as already discussed in Ref. 15.

A more careful analysis of the sampling limitation reveals that part of the reduction in the sensitivity of this technique comes from the use of a double sampling, one on the target that selects beamlets and one on the screen to image them. We have proposed and we are testing a new device, ${ }^{16}$ a sort of optical pepper pot, where there is only one sampling at the source level. It makes use of Optical Transition Radiation (OTR) produced when a charge passes through a metallic foil.

The angular distribution of the emerging radiation contains information about the angular divergence of the beam. Using an optical system to reproduce outside the vacuum chamber the source radiation field, and sampling by means of a microlens array, it is possible to measure the angular distribution and so the value of the beam divergence, in different transverse positions. It allows the retrievement of the correlation term. Acquiring at the same time also the beam spot allows, in principle, the measurement of the emittance in a single shot. In Fig. 3, there is a sketch of the system, with also a picture of the measurement to show the output of the method.

While we have already performed a test of this device, we were not able so far to retrieve the value of the emittance. The resolution is too poor at the actual SPARC_LAB ${ }^{17}$ energy value.

In fact, as it is shown in Fig. 4, due to the narrowing of the angular distribution at higher energy, the resolution of this kind of measurement increases with the energy. We consider as a reference for limiting visibility, a $10 \%$ value of the ratio intensity maximum/minimum in the angular distribution of the radiation.

Another single shot emittance measurement has been recently published ${ }^{18}$ relying on a method developed some years ago. ${ }^{19}$ An electron beam produce by LWFA is focused in a triplet of permanent magnets before entering in a dispersive dipole. Due to the large energy spread (usually several

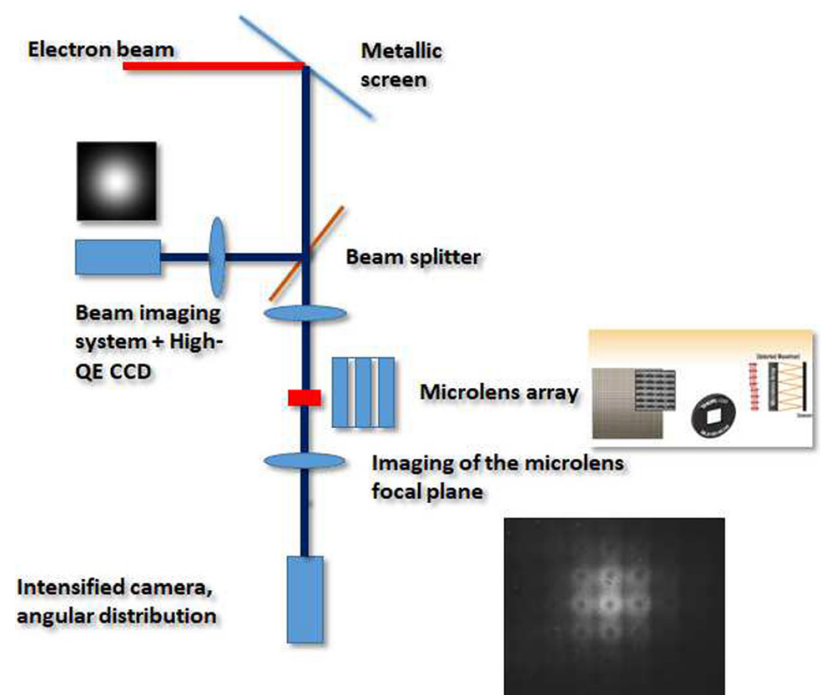

FIG. 3. The OTR produced by the beam is split into two arms. In one arm, a camera records the beam spot size. In the other arm, a replica of the radiation field on the source is produced in the front plane of a microlens array. The angular distribution is then retrieved for each microlens imaging their focal plane in an intensified camera. The experiment has been performed at the SPARC_LAB photoinjector at $125 \mathrm{MeV}$.

$\%$ in this kind of experiments), different energy parts inside the bunch are focused in different transverse dimensions. This system works very well for a large energy spread, but its main limit will appear in the case of energy spread on the order of $0.5 \%$ or lower, where the difference in focus could be really small.

\section{LONGITUDINAL DIAGNOSTICS}

While for transverse single shot measurement, there are only techniques still in developing, for longitudinal measurement, there are already several possibilities that can be implemented in our machine. Longitudinal diagnostics is mandatory to clearly set the correct compression phase in the velocity bunching and to recover the correlated energy spread induced in this way. We consider using different methods, tailoring the instrumentations to the particular machine condition. The

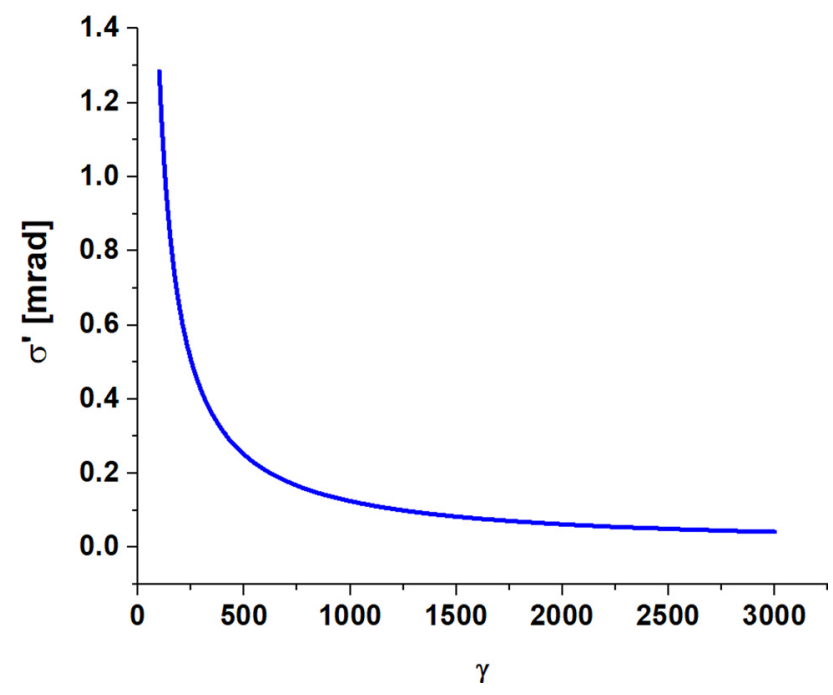

FIG. 4. Plot of the resolution in angle vs relativistic factor $\gamma$. 

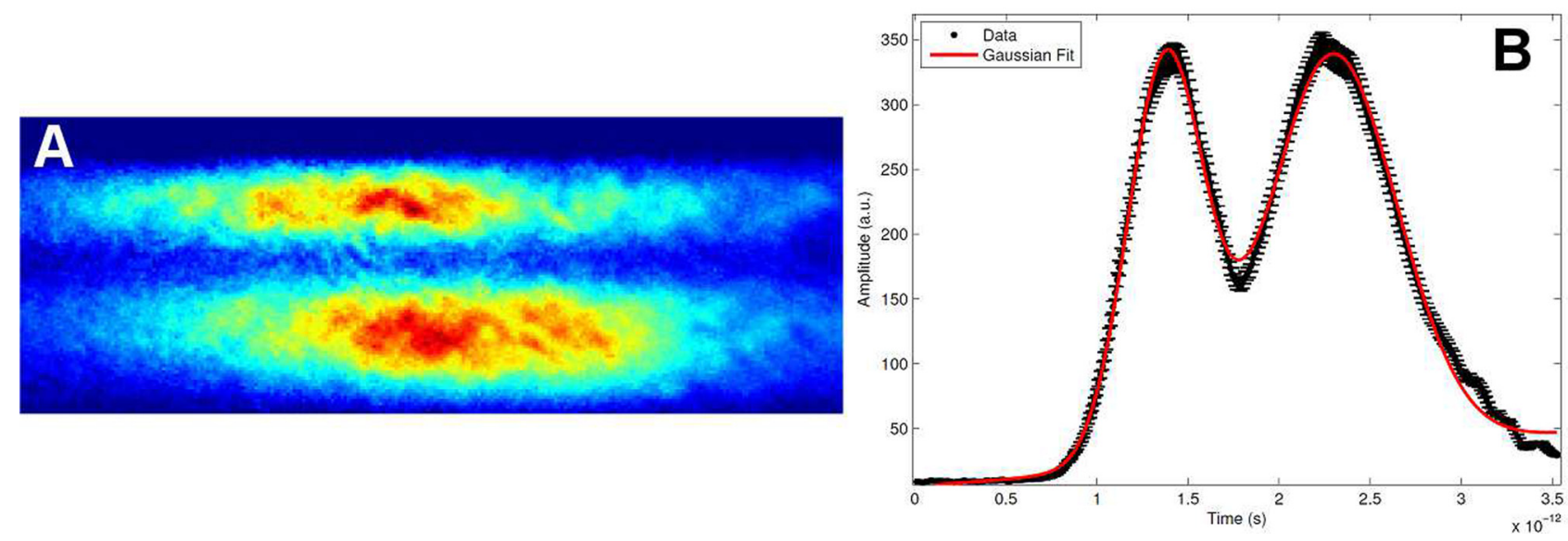

FIG. 5. (a) Image EOS of a bunch train of two bunches, $80 \mathrm{pC}$ on each bunch and pulse length $160 \mathrm{fs}$ and $200 \mathrm{fs}$, respectively. Their distance was $800 \mathrm{fs}$. The crystal is a GaP. (b) Profile of the EOS signal.

single shot longitudinal phase space measurement will be performed with an X-band RF deflector (RFD), i.e., a RF cavity with a transverse deflecting mode, combined with a magnetic dipole. The need of an X-band cavity is mainly due to the fs scale resolution obtainable in such a way. While this device can reach such a resolution, particular attention must be paid to its design. The reduced iris aperture and the possibility that the beam goes out of the center inside the device, due to the transverse field, must be considered with beam dynamics simulations. Only one X-band RFD operates so far at SLAC. ${ }^{20}$ It is designed for an energy one order of magnitude greater of our interesting case, so it could be used as a reference but it must be rescaled, at least in its length. Particular attention must be put also in the RF time jitter and the structure temperature stability, tailoring these requirements to the beam energy and the available beam line. As a rule of thumb, for the $\mathrm{GeV}$ level beam, a temperature stability on the order of tens of $\mathrm{mK}$ and time stability on the order of tens of femtoseconds are required.

However, for one shot not intercepting bunch length measurement, useful for instance when the beam is sent in a plasma module to correlate input and output properties of the bunches, two other systems must be implemented. Diffraction radiation ${ }^{21}$ is emitted when a charged particle passes through a hole with the transverse dimension smaller with respect to the radial extension of the electromagnetic field traveling with the charge. Coherent emission arises when the observed wavelength is longer with respect to the bunch length. ${ }^{22}$ For our case, where this time length can be in the range between few ps and few fs, it means to have several detectors, each one sensitive to a wide range of wavelengths ranging from far infrared (FIR) to visible light. This kind of measurement can be performed in the multi shot mode using a Martin Pupplet or Michelson interferometer, ${ }^{23}$ or in a single shot (highly desiderable) dispersing the radiation and collecting it in a linear detector. The complete analysis of the spectrum leads to the reconstruction of the longitudinal bunch shape. There are already several examples of such kind of measurements using different approaches. One is based on a single KRS-5 (thallium bromoiodide) prism, ${ }^{24}$ and another on a series of separate spectrometers working in different wavelength scales. ${ }^{25}$
Also, in order to set the compression phase, sometimes, only a relative measurement of the coherent radiation integrated on the whole bandwidth of the detector is enough. This system can be also used to monitor the phase stability of the section used for compression and to eventually stabilize it with a feedback.

Another single shot device is based on EOS (Electro Optical Sampling). The electric field co-propagating with the bunch can rotate the polarization of a laser impinging on a non linear crystal such as GaP or ZnTe.

Using the spatial decoding scheme ${ }^{26}$ realized with an angle of incidence between the probe laser and the crystal, it is possible to retrieve the longitudinal beam profile in one shot (Fig. 5). The advantage of such a scheme with respect to coherent radiation is definitely that there is no reconstruction of the bunch shape starting with frequency analysis, with the problems related to the correct transport and propagation of all the wavelengths in the spectrum. But the disadvantage is the temporal resolution, limited or by the crystal bandwidth or by the length of the laser probe. Typical values are on the order of 40-50 $\mathrm{fs} .{ }^{27}$ However, this diagnostics will be very important in our machine because while the $\mathrm{X}$ band RFD offers a high resolution for the measurement of very short bunches, i.e., in the fs region, it will be not the best choice for the ps bunch length. On the other end, the EOS can cover easily this range of dimensions, being also not intercepting. Also, it is often used as a bunch of arrival monitors, that it is very important in some plasma acceleration schemes, as for instance, external injection. ${ }^{28}$

\section{CONCLUSIONS}

The plasma acceleration, no matter which scheme is implemented, sets a series of challenges in all of the equipment, and in particular, in the diagnostics. Compact, single shot, high resolution devices must be foreseen to properly monitor the machine. In preparing a conceptual design report, we found that while there are already several diagnostics for longitudinal parameters that can be adapted to our case, there is a lack of techniques for single shot emittance. To overcome this problem, we propose a wide use of the betatron radiation to monitor the beam properties inside the 
plasma channel, and to test some new ideas outside the plasma, but only after capture optics, to measure the properties of the beam that will be used for applications.

\section{ACKNOWLEDGMENTS}

This work was supported by the European Union's Horizon 2020 research and innovation programme under Grant Agreement No. 653782.

${ }^{1}$ D. Wang, S. Antipov, C. Jing, J. Power, M. Conde, E. Wisniewski, W. Liu, J. Qiu, G. Ha, V. Dolgashev et al., "Interaction of an ultrarelativistic electron bunch train with a w-band accelerating structure: High power and high gradient," Phys. Rev. Lett. 116, 054801 (2016).

${ }^{2}$ G. Andonian, D. Stratakis, M. Babzien, S. Barber, M. Fedurin, E. Hemsing, K. Kusche, P. Muggli, B. O'Shea, X. Wei et al., "Dielectric wakefield acceleration of a relativistic electron beam in a slab-symmetric dielectric lined waveguide," Phys. Rev. Lett. 108, 244801 (2012).

${ }^{3}$ C. Gahn, G. Tsakiris, A. Pukhov, J. Meyer-ter Vehn, G. Pretzler, P. Thirolf, D. Habs, and K. Witte, "Multi-MeV electron beam generation by direct laser acceleration in high-density plasma channels," Phys. Rev. Lett. 83, 4772 (1999)

${ }^{4}$ P. A. Walker, P. Alesini, A. Alexandrova, M. P. Anania, N. Andreev, I. Andriyash, A. Aschikhin, R. Assmann, T. Audet, A. Bacci et al., "Horizon 2020 EuPRAXIA design study," J. Phys.: Conf. Ser. 874, 012029 (2017).

${ }^{5} \mathrm{~W}$. Graves and E. Johnson, "A high resolution electron beam profile monitor," in Proceedings of the 1997 Particle Accelerator Conference, 1997 (IEEE, 1997), Vol. 2, pp. 1993-1995.

${ }^{6}$ F. Löhl, S. Schreiber, M. Castellano, G. Di Pirro, L. Catani, A. Cianchi, and K. Honkavaara, "Measurements of the transverse emittance at the flash injector at DESY," Phys. Rev. Spec. Top.-Accel. Beams 9, 092802 (2006). ${ }^{7}$ M. Migliorati, A. Bacci, C. Benedetti, E. Chiadroni, M. Ferrario, A. Mostacci, L. Palumbo, A. Rossi, L. Serafini, and P. Antici, "Intrinsic normalized emittance growth in laser-driven electron accelerators," Phys. Rev. Spec. Top.-Accel. Beams 16, 011302 (2013).

${ }^{8}$ M. R. Conti et al., "Electron beam transfer line design for plasma driven free electron lasers," Nucl. Instrum. Methods Phys. Res., Sect. A (in press).

${ }^{9}$ A. Rousse, K. T. Phuoc, R. Shah, A. Pukhov, E. Lefebvre, V. Malka, S. Kiselev, F. Burgy, J.-P. Rousseau, D. Umstadter et al., "Production of a $\mathrm{keV} \mathrm{x}$-ray beam from synchrotron radiation in relativistic laser-plasma interaction," Phys. Rev. Lett. 93, 135005 (2004).

${ }^{10}$ G. Plateau, C. Geddes, D. Thorn, M. Chen, C. Benedetti, E. Esarey, A. Gonsalves, N. Matlis, K. Nakamura, C. Schroeder et al., "Low-emittance electron bunches from a laser-plasma accelerator measured using singleshot X-ray spectroscopy," Phys. Rev. Lett. 109, 064802 (2012).

${ }^{11}$ S. Kneip, C. McGuffey, J. Martins, M. Bloom, V. Chvykov, F. Dollar, R. Fonseca, S. Jolly, G. Kalintchenko, K. Krushelnick et al., "Characterization of transverse beam emittance of electrons from a laserplasma wakefield accelerator in the bubble regime using betatron x-ray radiation," Phys. Rev. Spec. Top.-Accel. Beams 15, 021302 (2012).

${ }^{12}$ A. Curcio, M. Anania, F. Bisesto, E. Chiadroni, A. Cianchi, M. Ferrario, F. Filippi, D. Giulietti, A. Marocchino, M. Petrarca et al., "Trace-space reconstruction of low-emittance electron beams through betatron radiation in laser-plasma accelerators," Phys. Rev. Accel. Beams 20, 012801 (2017).

${ }^{13}$ A. Curcio, M. Anania, F. Bisesto, E. Chiadroni, A. Cianchi, M. Ferrario, F. Filippi, D. Giulietti, A. Marocchino, F. Mira et al., "Single-shot non-intercepting profile monitor of plasma-accelerated electron beams with nanometric resolution,” Appl. Phys. Lett. 111, 133105 (2017).

${ }^{14} \mathrm{C}$. Lejeune and J. Aubert, "Emittance and brightness: Definitions and measurements," in Applied Charged Particle Optics (Academic Press, New York, 1980).

${ }^{15}$ A. Cianchi, M. Anania, M. Bellaveglia, M. Castellano, E. Chiadroni, M. Ferrario, G. Gatti, B. Marchetti, A. Mostacci, R. Pompili et al., "Challenges in plasma and laser wakefield accelerated beams diagnostic," Nucl. Instrum. Methods Phys. Res., Sect. A 720, 153-156 (2013).

${ }^{16}$ A. Cianchi, M. Anania, M. Bellaveglia, F. Bisesto, M. Castellano, E. Chiadroni, D. Di Giovenale, G. Di Pirro, M. Ferrario, G. Gatti et al., "Transverse emittance diagnostics for high brightness electron beams," Nucl. Instrum. Methods Phys. Res., Sect. A 865, 63-66 (2017).

${ }^{17}$ M. Ferrario, D. Alesini, M. Anania, A. Bacci, M. Bellaveglia, O. Bogdanov, R. Boni, M. Castellano, E. Chiadroni, A. Cianchi et al., "SPARC_LAB present and future," Nucl. Instrum. Methods Phys. Res., Sect. B 309, 183-188 (2013).

${ }^{18}$ S. Barber, J. van Tilborg, C. Schroeder, R. Lehe, H.-E. Tsai, K. Swanson, S. Steinke, K. Nakamura, C. Geddes, C. Benedetti et al., "Measured emittance dependence on the injection method in laser plasma accelerators," Phys. Rev. Lett. 119, 104801 (2017).

${ }^{19}$ R. Weingartner, S. Raith, A. Popp, S. Chou, J. Wenz, K. Khrennikov, M. Heigoldt, A. R. Maier, N. Kajumba, M. Fuchs et al., "Ultralow emittance electron beams from a laser-wakefield accelerator," Phys. Rev. Spec. Top.-Accel. Beams 15, 111302 (2012).

${ }^{20}$ V. A. Dolgashev and J. Wang, "RF design of X-band rf deflector for femtosecond diagnostics of LCLS electron beam," AIP Conf. Proc. 1507, 682-687 (2012).

${ }^{21}$ M. L. Ter-Mikaelian, High Energy Electromagnetic Processes in Condensed Media (Wiley, New York, 1972).

${ }^{22}$ M. Castellano, V. Verzilov, L. Catani, A. Cianchi, G. Orlandi, and M. Geitz, "Measurements of coherent diffraction radiation and its application for bunch length diagnostics in particle accelerators," Phys. Rev. E 63, 056501 (2001).

${ }^{23}$ E. Chiadroni, M. Bellaveglia, P. Calvani, M. Castellano, L. Catani, A. Cianchi, G. Di Pirro, M. Ferrario, G. Gatti, O. Limaj et al., "Characterization of the $\mathrm{THz}$ radiation source at the Frascati linear accelerator," Rev. Sci. Instrum. 84, 022703 (2013).

${ }^{24}$ T. Maxwell, C. Behrens, Y. Ding, A. Fisher, J. Frisch, Z. Huang, and H. Loos, "Coherent-radiation spectroscopy of few-femtosecond electron bunches using a middle-infrared prism spectrometer," Phys. Rev. Lett. 111, 184801 (2013).

${ }^{25}$ M. Heigoldt, A. Popp, K. Khrennikov, J. Wenz, S.-W. Chou, S. Karsch, S. Bajlekov, S. Hooker, and B. Schmidt, "Temporal evolution of longitudinal bunch profile in a laser wakefield accelerator," Phys. Rev. Spec. Top.Accel. Beams 18, 121302 (2015).

${ }^{26}$ R. Pompili, A. Cianchi, D. Alesini, M. Anania, A. Bacci, M. Bellaveglia, M. Castellano, E. Chiadroni, D. D. Giovenale, G. D. Pirro et al., "First single-shot and non-intercepting longitudinal bunch diagnostics for comblike beam by means of electro-optic sampling," Nucl. Instrum. Methods Phys. Res., Sect. A 740, 216-221 (2014).

${ }^{27}$ B. Steffen, V. Arsov, G. Berden, W. A. Gillespie, S. Jamison, A. M. MacLeod, A. van der Meer, P. Phillips, H. Schlarb, B. Schmidt et al., "Electro-optic time profile monitors for femtosecond electron bunches at the soft x-ray free-electron laser flash," Phys. Rev. Spec. Top.-Accel. Beams 12, 032802 (2009).

${ }^{28}$ C. Clayton and L. Serafini, "Generation and transport of ultrashort phaselocked electron bunches to a plasma beatwave accelerator," IEEE Trans. Plasma Sci. 24, 400-408 (1996). 